\title{
Hunting down heterogeneity
}

\author{
The search for mechanisms and meaning within structurally and functionally diverse systems \\ requires different expectations and approaches.
}

Heterogeneity has the potential to be overwhelming. Variations in form, function and behavior exist everywhere-in every cell, tissue and organism. Some biomolecules are famous for their structural diversity, as is the case for antibodies and heparin sulfate. The diversity of others is only beginning to be realized, as in the expanding catalog of post-translational and epigenetic modifications of proteins and DNA. Beyond this growing library of structures, improved technological capabilities are exposing the functional variation that occurs in biological systems with increasing precision, with the result that what was once thought of as error in a measurement can often now be ascribed to distinct conformations and populations. Understanding the scope and consequences of these new discoveries will require that biological hypotheses be framed and tested in new ways. Toward this end, we present a collection of articles focusing on the origins, analysis and outcomes of heterogeneity.

Where does heterogeneity arise? Structural variations can result from errors in building a specified product, such as in the misincorporation of amino acids during translation. Similarly, enzyme reactions may be interrupted or promiscuous, yielding incomplete products or heterogeneous pools of products. Fincher and colleagues (Review, p. 724) describe how some enzymes, such as the glycosyltransferases that construct plant cell walls, synthesize polymers without a template, yielding heterogeneity by design. Structural differences can serve to create heterogeneity on larger scales, as in the altered localization of differentially modified biomolecules or the stochastic behavior of genes.

Scientists are increasingly mindful of these kinds of variations in their ongoing research. However, this changing mindset must also be reflected backward to reconsider known molecules and processes, looking for information that might have been missed. For example, advanced analytical tools are providing compelling evidence in some cases that what we have traditionally thought of as a single, homogeneous biomolecule can be composed of several different forms of very similar molecules that may or may not act in a unified manner. This is especially true for glycoproteins, where it is increasingly understood that glycans play significant roles in protein folding and function. As Rudd and colleagues discuss (Review, p. 713), defining the specific glycans present-much less determining those responsible for a desired function-is complicated; this has similarly complicated the development and approval of glycoproteins as biological therapies (Chem. \& Eng. News 87, 20-23, 2009).

Why is it so hard to characterize heterogeneity? We can be limited by materials, available technologies and even our own expectations. Improvements in techniques such as mass spectrometry, microfluidics and fluorescence spectrometry have pushed the limits of resolution to single cells and single proteins, but these methods are not applicable to all problems. Additionally, we may not know the full extent of diversity in any given system. In these cases, Puskas cautions that scientists' preconceptions can affect their analysis of the information at hand (Elements, p. 697). Finally, it is difficult to know a priori which biological systems are most relevant for study, in part because heterogeneity can be masked when different mechanisms lead to similar outcomes. For example, a multitude of mutations affecting numerous cellular pathways lead generically to cancer. Developing more sophisticated techniques and nomenclature to identify different mutations, protein isoforms or bacterial growth phenotypes (Research Highlights, p. 698) can better describe the true complexity present.

On top of these considerations, heterogeneity itself is heterogeneous. Thus, the differences observed in one cell or one protein can be different in another environment. In these cases, scientists must carefully weigh the insight that may be gained from cataloging these changes against the significant time commitment that may be required for the complete enumeration of a system's variability. Indeed, much research proceeds by measuring averages, including averages of enzyme rates, metabolite concentrations and the timing of cell cycle checkpoints. In some cases, our initial understanding as defined by these averages has turned out to be surprisingly general across length scales and conditions, as, for example, in comparing ensemble measurements to the function of single enzymes in vitro
(Nat. Chem. Biol. 2, 87-94, 2006) or in cells (Nat. Chem. Biol. 6, 485-487, 2010). In other cases, the 'average' becomes meaningless when investigated in more detail, yielding entirely new interpretations of a known biological readout.

What are the functional consequences of heterogeneity? Tawfik (Commentary, p. 692) describes "biological messiness" as the raw material for evolution and adaptation. Indeed, Lidstrom and Konopka (Review, p. 705) highlight our increasing understanding of diversity in microbial populations as a driver for bacterial robustness and resistance. At the cellular level, structural diversity creates a dense network of behaviors. For example, the assembly of different $G$ protein-coupled receptors into diverse heteromers can connect to different intracellular pathways. For heparin sulfate, different extents of sulfation result in different binding affinities to a variety of biological targets, thus altering their functional readout. In contrast, for spider silk and other elastomers, variations in protein composition may simply but significantly alter the physical properties of the aggregate (News \& Views, p. 702). Unfortunately, ascribing function to specific molecules or cellular subpopulations is complicated by the fact that functions are rarely an all-or-nothing response, and thus a single functional readout can depend on the collective action of a suite of molecules. However, careful experimental design and thoughtful analysis can often bring meaning to the messiness.

Chemical biologists are particularly well suited to tackle these messy topics, as many of these questions require careful consideration of structures and mechanisms at the molecular level. Additionally, chemical biologists are apt toolmakers, not just through improvements to existing technologies but through the development of orthogonal and/or creative strategies that fill gaps in our arsenal of techniques (p. 733 p. 750; Nat. Chem. Biol. 6, 645-651, 2010). Finally, scientists who are or can quickly become conversant in new fields can both provide the impetus needed to re-evaluate current thinking and share strategies across disciplines for maximum benefit. An increased understanding of heterogeneity, made possible by new tools, techniques and theories, will transform our appreciation for the complexity of life. 